\title{
Size-Based Characterization of Adalimumab and TNF-a Interactions Using Flow Induced Dispersion Analysis: Assessment of Avidity-Stabilized Multiple Bound Species
}

Henrik Jensen ( $\boldsymbol{Q}$ henrik@fidabio.com )

FIDA Biosystems Aps

Morten Pedersen

FIDA Biosystems Aps

Jesper Østergaard

University of Copenhagen

Ragna Haegebaert

University of Copenhagen

\section{Research Article}

Keywords: biomolecular, FIDA, hydrodynamic

Posted Date: December 1st, 2020

DOl: https://doi.org/10.21203/rs.3.rs-113718/v1

License: (c) (1) This work is licensed under a Creative Commons Attribution 4.0 International License.

Read Full License

Version of Record: A version of this preprint was published at Scientific Reports on February 26th, 2021. See the published version at https://doi.org/10.1038/s41598-021-84113-z. 


\title{
Size-based characterization of
}

\section{adalimumab and TNF- $\alpha$ interactions}

\section{using Flow Induced Dispersion Analysis:}

\author{
Assessment of Avidity-stabilized \\ Multiple Bound Species
}

Morten E. Pedersen ${ }^{\dagger,} \mathrm{a}, \mathrm{b}$, Ragna M.S. Haegebaert ${ }^{\dagger, \mathrm{b}}$, Jesper Østergaard ${ }^{\mathrm{b}}$, and $\underline{\text { Henrik Jensen }{ }^{\mathrm{a}, \mathrm{b}^{*}}}$

${ }^{a}$ Fida Biosystems ApS, Fruebjergvej 3, 2100 Copenhagen O, Denmark.

${ }^{b}$ Department of Pharmacy, University of Copenhagen, Universitetsparken 2, 2100 Copenhagen O, Denmark.

$\dagger$ These authors contributed equally

*Corresponding author: Henrik Jensen

Email: henrik@fidabio.com 


\begin{abstract}
The understanding and characterization of protein interactions is crucial for elucidation of complicated biomolecular processes as well as for the development of new biopharmaceutical therapies. Often, protein interactions involve multiple binding, avidity, oligomerization, and are dependent on the local environment. Current analytical methodologies are unable to provide a detailed mechanistic characterization considering all these parameters, since they often rely on surface immobilization, cannot measure under biorelevant conditions, or do not feature a structurally-related readout for indicating formation of multiple bound species.

In this work, we report the use of flow induced dispersion analysis (FIDA) for in-solution characterization of complex protein interactions under in-vivo like conditions. FIDA is an immobilization-free ligand binding methodology employing Taylor dispersion analysis for measuring the hydrodynamic radius (size) of biomolecular complexes. Here, the FIDA technology is utilized for a size-based characterization of the interaction between TNF- $\alpha$ and adalimumab. We report concentration-dependent complex sizes, binding affinities $\left(K_{\mathrm{d}}\right)$, kinetics, and higher order stoichiometries, thus providing essential information on the TNF- $\alpha$ adalimumab binding mechanism. Furthermore, it is shown that the avidity stabilized complexes involving formation of multiple non-covalent bonds are formed on a longer timescale than the primary complexes formed in a simple 1 to 1 binding event.
\end{abstract}




\section{Introduction}

The elucidation of biomolecular interactions is key to understanding physiological processes on a molecular level as well as for developing novel pharmaceutical therapies ${ }^{1-5}$. Hence, appropriate analytical methodologies are required in order to comprehensively characterize and quantify these interactions ${ }^{4-7}$. This is often achieved via functional assays such as ligand binding assays (LBA) and cell-based assays, that serve as an important surrogate of the anticipated biological mechanism in patients ${ }^{4-7}$. Ligand binding technologies are capable of reporting apparent binding affinities, but most methods lack a detailed deconvolution on the structural mode of action ${ }^{6,8}$. Often the relevant interactions involve higher order stoichiometries driven by avidity and oligomerization; rarely a simple 1:1 binding model provides a full picture of the underlying biology $3,9,10$. For example, the oligomeric state of a protein may determine whether it is pathological or not, as seen with tumor necrosis alpha (TNF- $\alpha$ ) where the TNF- $\alpha$ monomer and trimer are inactive and active inflammatory cytokines, respectively ${ }^{11}$. It is therefore often necessary to combine LBAs with structural methods (e.g. size-exclusion chromatography, SDSPAGE, mass spectrometry) to get the full picture ${ }^{4-7}$. However, structural methodologies require relatively clean samples, which may not reflect true in-vivo conditions ${ }^{12}$. Ideally, functional assays should report an absolute measure of affinity in combination with structural information under biorelevant conditions.

Flow Induced Dispersion Analysis (FIDA) is a novel analytical platform for size-based characterization of biomolecules including assessment of binding affinity $\left(K_{\mathrm{d}}\right)$, complex hydrodynamic radius, analyte quantification, and physical stability ${ }^{13-17}$. The FIDA principle relies on Taylor dispersion analysis (TDA) for measuring the apparent hydrodynamic radius (size) of a selective ligand (the indicator) as it binds to the analyte (e.g., the protein/biomolecule) of interest $^{13}$. The apparent size of the indicator is measured in a titration series with varying analyte concentrations. The resulting binding curve is used for determining the binding affinity $\left(K_{\mathrm{d}}\right)$ and complex size. FIDA is highly tolerant to analysis in complex and crude sample matrices, 
such as plasma and fermentation broth $15,17,18$, and the absolute size readout provides information on structural features of the formed complex as well as avidity stabilization of multiple bound species.

Adalimumab is a recombinant monoclonal IgG antibody, frequently used in the treatment of auto-immune diseases such as rheumatoid arthritis and Crohn's disease ${ }^{9,19}$. It binds and neutralizes the inflammatory cytokine TNF- $\alpha$ with high affinity and specificity ${ }^{9,19}$. The interaction between TNF- $\alpha$ and adalimumab has been characterized applying various techniques such as surface plasmon resonance (SPR), bio-layer interferometry (BLI), isothermal titration calorimetry (ITC), electron microscopy (EM), dynamic light scattering (DLS), analytical ultracentrifugation and HPLC-based procedures 10,20-24. However, under physiological conditions TNF- $\alpha$ is a trimer with up to 3 binding sites and adalimumab has two binding sites which may make characterization of the TNF- $\alpha$ - adalimumab interaction challenging. In particular, surfacebased methodologies lack the possibility of easily controlling the concentrations of both binding partners, which complicates quantitative assessment of avidity effects. For instance, the TNF- $\alpha$ adalimumab interaction was recently characterized using hydrodynamic friction measurements ${ }^{25}$, which confirmed strong avidity effects and the presence of higher order complexes. The existing studies of the TNF- $\alpha$ - adalimumab system have been primarily conducted in neat buffer solutions, hence, little is known on TNF- $\alpha$ - adalimumab interactions under native (in-solution) and biorelevant conditions (e.g., in human plasma).

In this work, we have developed FIDA assays for size-based characterization of the interaction between trimeric TNF- $\alpha$ and adalimumab in buffer as well as human plasma. The assays were based on TNF- $\alpha$ as the indicator (ligand); hence, the apparent size change of TNF- $\alpha$ was measured in the presence of varying adalimumab concentrations. We report the in-solution binding affinity $\left(K_{\mathrm{d}}\right)$ and avidity stabilized complexes, size of the formed complexes, stoichiometry modeling, and qualitative assessment of the kinetics involved in formation of lower and higher order complexes. 


\section{Results and discussion}

\section{Characterization of TNF- $\alpha$ and adalimumab interactions in pre-incubated}

\section{buffer samples}

Characterization of biomolecular interactions using FIDA is based on an indicator molecule, TNF- $\alpha$, selectively interacting with the analyte of interest, adalimumab. Initially, the hydrodynamic radius (size) of TNF- $\alpha$ is measured in the absence of adalimumab, thus the size of unbound TNF- $\alpha$ is measured confirming the structural integrity of the molecule. As the concentration of adalimumab is increased, the apparent size of TNF- $\alpha$ increases due to a higher degree of binding. Full details on how to attain the hydrodynamic radius of the indicator from the raw data, i.e. Taylorgrams, has been described elsewhere ${ }^{14,17}$. The apparent TNF- $\alpha$ size is plotted as a function of adalimumab concentration, thereby providing a means for in-solution characterization of affinity $\left(K_{\mathrm{d}}\right)$, stoichiometry, and complex size.

The hydrodynamic radii of unbound TNF- $\alpha$-alexa488 (100 nM) and adalimumab $(1 \mathrm{mg} / \mathrm{mL})$ were determined by TDA to $3.20 \pm 0.04 \mathrm{~nm}$ and $5.6 \pm 0.24 \mathrm{~nm}$, respectively. The size of TNF- $\alpha$ confirmed the expected oligomeric state as trimeric at physiological pH 7.4. Kohno et al. reported a hydrodynamic radius of $3.1 \mathrm{~nm}$ for the trimer using SEC-LC at the same $\mathrm{pH}^{20}$, and Daub et al. found the structure of TNF- $\alpha$ as trimeric at $100 \mathrm{nM}(\mathrm{pH}$ 7.4) using hydrodynamic friction measurements ${ }^{25}$. The determined hydrodynamic radius of adalimumab was consistent with previous TDA measurements $(5.6-5.9 \mathrm{~nm})^{26}$. 


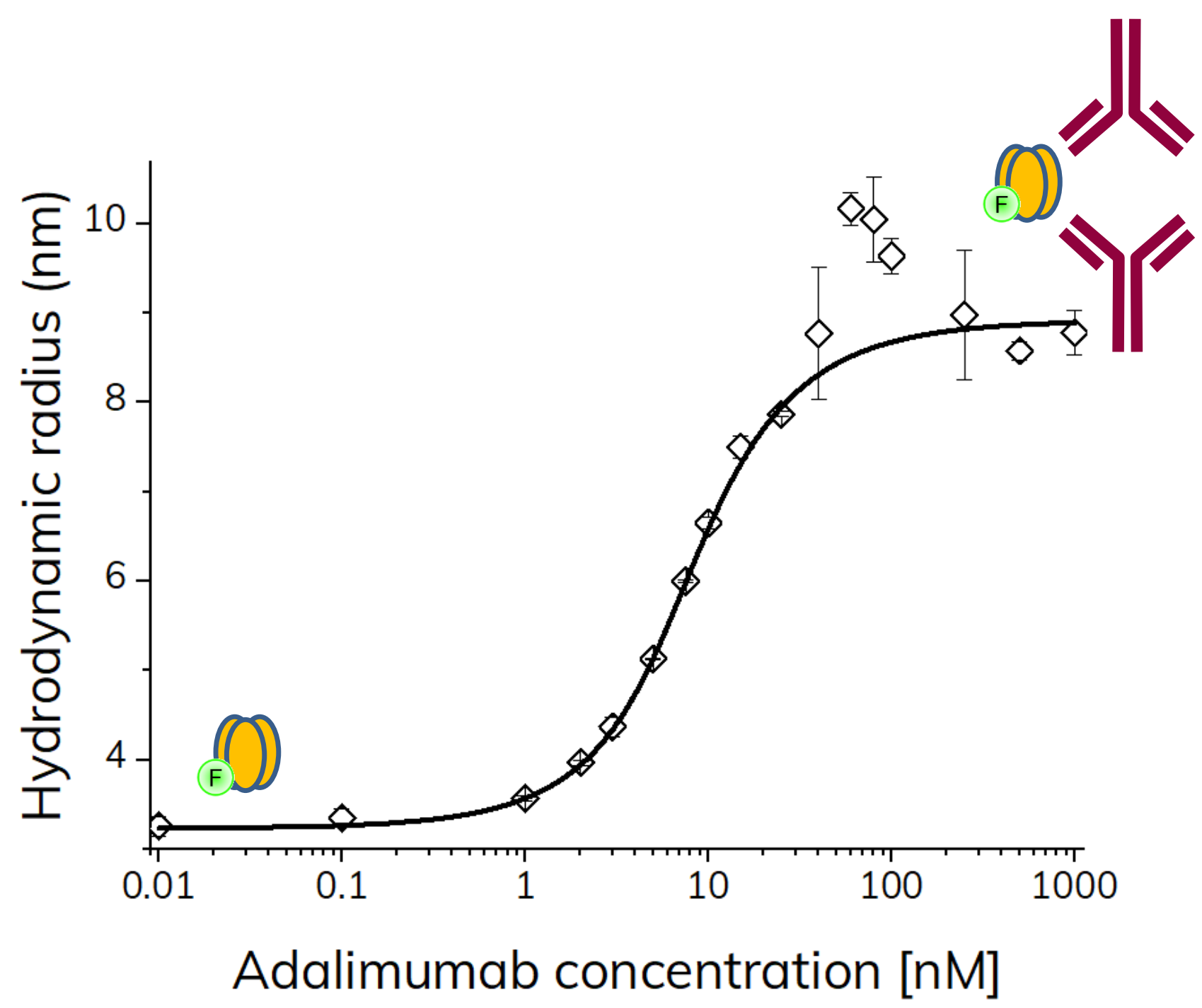

Figure 1: Apparent hydrodynamic radius of TNF- $\alpha$-alexa488 (100 $\mathrm{nM}$ ) as function of adalimumab concentration (0 - $1000 \mathrm{nM}$ ) determined by FIDA in $67 \mathrm{mM}$ phosphate buffer with $0.1 \% \mathrm{v} / \mathrm{v}$ HSA at pH 7.40 and $25^{\circ} \mathrm{C}$ using pre-incubated samples $(n=3$, error bars represent standard deviation). The solid line shows fitting to the excess indicator binding isotherm (equation 7).

Subsequently, the apparent size of TNF- $\alpha$-alexa488 (100 nM) was measured throughout an adalimumab titration series $(0-1000 \mathrm{nM})$ in assay buffer containing $0.1 \% \mathrm{v} / \mathrm{v} \mathrm{HSA}$, which was added in order to minimize surface adsorption of TNF- $\alpha$ to glass vials and pipette tips. The apparent size of TNF- $\alpha$ increased with increasing adalimumab concentrations resulting in a binding curve (Fig. 1). The increase in apparent size was due to the formation of TNF- $\alpha$ adalimumab complexes. At 60-120 nM adalimumab, the binding curve exhibited apparent complex sizes up to $10 \mathrm{~nm}$, which declined to around $8.5 \mathrm{~nm}$ with adalimumab concentrations above $120 \mathrm{nM}$. This led to a hook-shaped binding curve (Fig. 1) indicating that a simple 1:1 binding does not apply to the TNF- $\alpha$-adalimumab interaction and that larger complexes are formed under certain conditions. Trimeric TNF- $\alpha$ possesses three binding sites and adalimumab 
is an antibody with two binding sites, hence allowing for formation of various binding stoichiometries. The formation of higher order complexes is favored when the concentrations of TNF- $\alpha$ and adalimumab are nearly similar, as seen in Fig. 1. This is in agreement with previous findings where different complex stoichiometries have been observed ${ }^{20-23}$. The concentrations and formation of higher order complexes are heavily governed by the absolute and relative concentrations of the interacting species. Thus, at excess adalimumab concentrations (500-1000 nM, Fig. 1), the complex formation was shifted towards smaller species. This was also observed in a study using analytical ultracentrifugation and DLS22.

The simple 1:1 excess binding model (equation 7) appeared to fit the data well (Fig. 1, solid line), except for concentrations where higher order structures were favored, resulting in an apparent $K_{\mathrm{d}}$ of $1.52 \mathrm{nM}$ and complex size of $8.9 \mathrm{~nm}\left(\mathrm{R}^{2}: 0.992\right)$.

Collectively, these results confirmed the oligomeric state of TNF- $\alpha$-alexa488 as trimeric from the size measurement of unbound TNF- $\alpha$ as well as its ability to form higher order complexes with adalimumab. Also, an apparent 1:1 $K_{\mathrm{d}}$ was calculated for the TNF- $\alpha$-adalimumab interaction. However, a more advanced binding model is needed to further quantify the formation of higher order complexes, as well as taking avidity effects into account.

\section{Extended binding model of TNF- $\alpha$ and adalimumab interactions in pre- incubated buffer samples}

A range of different TNF- $\alpha$-adalimumab stoichiometries, e.g., 1:1, 1:2, 2:1, 2:2, 3:2, has been reported ${ }^{20-23}$. On this basis, we set out to derive an extended binding model to describe the data set in Fig. 1. The binding scheme below is suggested to represent a better description of the TNF$\alpha$-adalimumab interactions considering the observed higher order structures:

$\mathrm{TA} \rightleftharpoons \mathrm{T}+\mathrm{A} \quad K_{\mathrm{d}_{1}}$ 


$$
\begin{array}{ll}
\mathrm{T}_{2} \mathrm{~A} \rightleftharpoons \mathrm{TA}+\mathrm{T} & K_{\mathrm{d}_{2}} \\
\mathrm{TA}_{2} \rightleftharpoons \mathrm{TA}+\mathrm{A} & K_{\mathrm{d}_{3}} \\
(\mathrm{TA})_{2} \rightleftharpoons \mathrm{TA}+\mathrm{TA} & K_{\mathrm{d}_{4}} \\
(\mathrm{TA})_{2} \rightleftharpoons \mathrm{T}_{2} \mathrm{~A}+\mathrm{A} & K_{\mathrm{d}_{5}} \\
(\mathrm{TA})_{2} \rightleftharpoons \mathrm{TA}_{2}+\mathrm{T} & K_{\mathrm{d}_{6}}
\end{array}
$$

where $\mathrm{T}$ and A represent trimeric TNF- $\alpha$ and adalimumab, respectively. The formation of a 2:2 (TNF- $\alpha$ :adalimumab) complex $\left(K_{\mathrm{d}_{4}}, K_{\mathrm{d}_{5}}\right.$ and $\left.K_{\mathrm{d}_{6}}\right)$ is expected to occur to a lesser extent than 1:1 $\left(K_{\mathrm{d}_{1}}\right), 2: 1\left(K_{\mathrm{d}_{2}}\right), 1: 2\left(K_{\mathrm{d}_{3}}\right)$ complexes as it involves multiple interactions leading to a substantial avidity effect. The apparent hydrodynamic radius measured is considered to be a weighted average of the hydrodynamic radii of $\mathrm{T}, \mathrm{TA}, \mathrm{TA}_{2}$ and $(\mathrm{TA})_{2}$. Additional higher order structures, such as $T_{2} A_{3}$ and $T A_{3}$, have also been reported ${ }^{22,23}$, but were not taken into account here because the used concentrations of TNF- $\alpha$ and adalimumab would most likely not favor these complexes. The binding scheme listed above can be simulated leading to estimates of the six $K_{d}$ 's, as shown in the Supplementary Information. It is assumed that $K_{\mathrm{d}_{1}}, K_{\mathrm{d}_{2}}$ and $K_{\mathrm{d}_{3}}$ as well as $K_{\mathrm{d}_{4}}, K_{\mathrm{d}_{5}}$ and $K_{\mathrm{d}_{6}}$ are identical. In our simulation, two data sets were used corresponding to 10 and $100 \mathrm{nM}$ TNF- $\alpha$ (Fig. 2). The dispersion of TNF- $\alpha$ inside the capillary during FIDA analysis results in a dilution effect, thus the total TNF- $\alpha$ concentration was set to obtain the best possible fit (see Supplementary Information). The simulation parameters, i.e., hydrodynamic radii and $K_{\mathrm{d}}$ values in Table 1 were estimated according to the findings in Fig. 1 as well as to the ability of the model to fit the experimental data. The $K_{\mathrm{d}_{4}}, K_{\mathrm{d}_{5}}$ and $K_{\mathrm{d}_{6}}$ are avidity stabilized (two non-covalent bonds are formed in the binding event), thus the values are approximately an order of magnitude lower. 
Table 1. Simulation parameters used to obtain the simulated curves at 10 and $100 \mathrm{nM}$ TNF- $\alpha$.

\begin{tabular}{|c|c|c|c|c|c|c|c|c|c|c|}
\hline \multicolumn{4}{|c|}{$\begin{array}{c}\text { Complex size reported as } \\
\text { hydrodynamic radius (nm) }\end{array}$} & \multicolumn{6}{c|}{ Dissociation constant } \\
(nM)
\end{tabular}

The model and selected simulation parameters seem to explain and fit the experimental data well, including the hook shape of the binding curves (Fig. 2). At low concentrations of adalimumab, TNF- $\alpha$ is the dominant species in solution whereas at high concentrations of adalimumab the dominant species is the $\mathrm{TA}_{2}$ complex.

a)

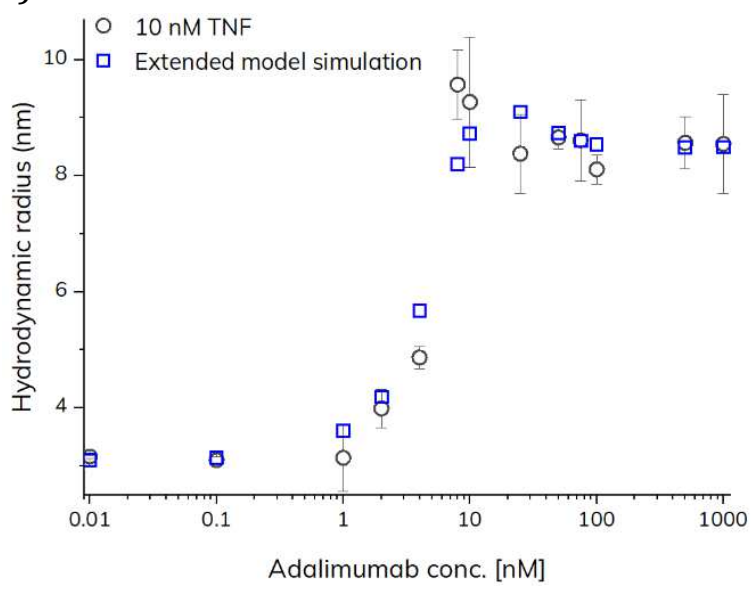

b)

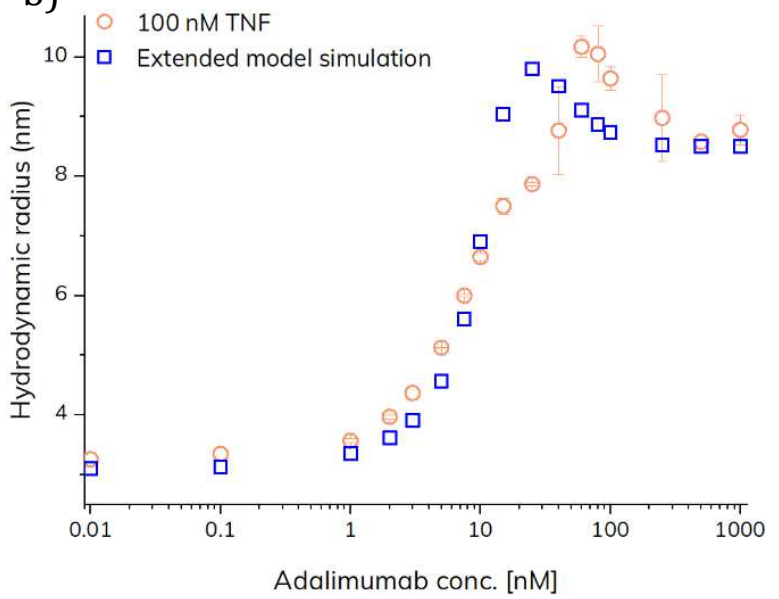

Figure 2: Extended model simulations of 10 (a) and 100 (b) $n M$ TNF- $\alpha$-alexa488 (open squares) as function of adalimumab concentration ( 0 - $1000 \mathrm{nM}$ ) compared to FIDA measurements in $67 \mathrm{mM}$ phosphate buffer with $0.1 \% \mathrm{v} / \mathrm{v} H S A$ at $p H 7.40$ and $25^{\circ} \mathrm{C}$ using pre-incubated samples (open circles, $n=3$, error bars represent standard deviation).

Interestingly, the amounts of the different species were heavily dependent on both the absolute and relative concentrations of TNF- $\alpha$ and adalimumab. Figure 3 shows how the fractions of the different species varies at a fixed TNF- $\alpha$ concentration of $100 \mathrm{nM}$ in the pre-incubated samples. Under these conditions the large $(\mathrm{TA})_{2}$ complex is the dominant species at intermediary adalimumab concentrations ( $\sim 10 \mathrm{nM})$, as expected from the experimental data (Fig. 2b). 


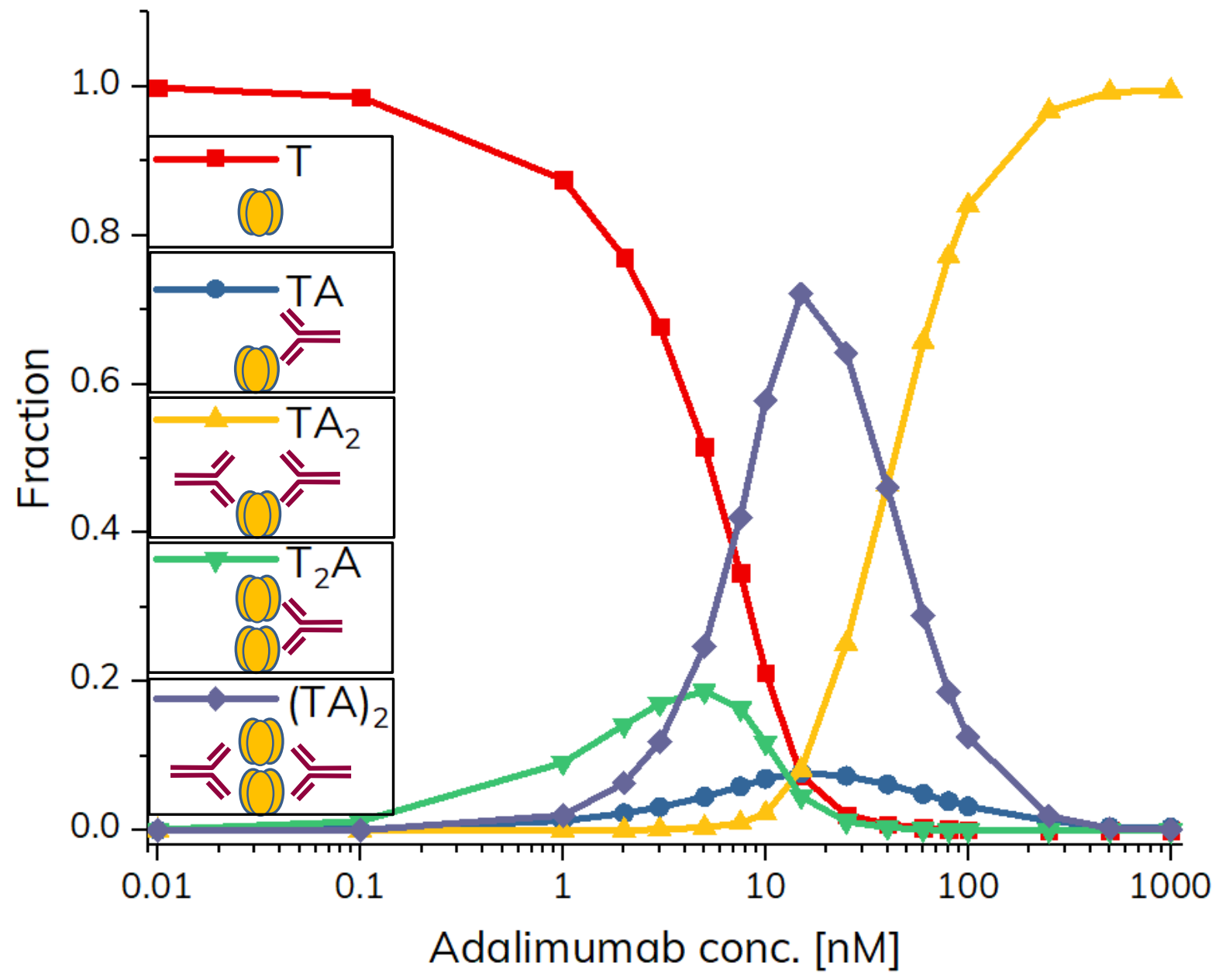

Figure 3: Simulated fractions of the various complexes between TNF- $\alpha$ and adalimumab as a function of adalimumab concentration corresponding to the experimental dataset at $100 \mathrm{nM}$ TNF- $\alpha$ in the pre-incubated samples. Data points are based on the extended model simulations.

a)

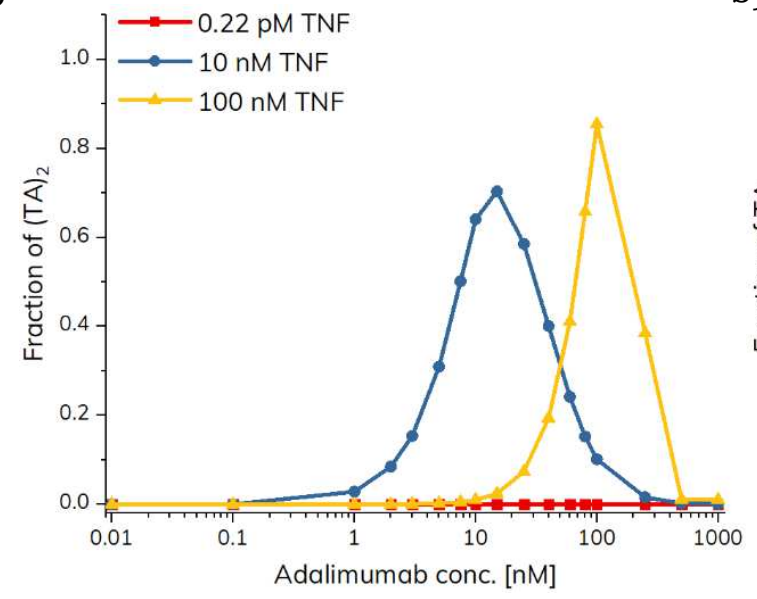

b)

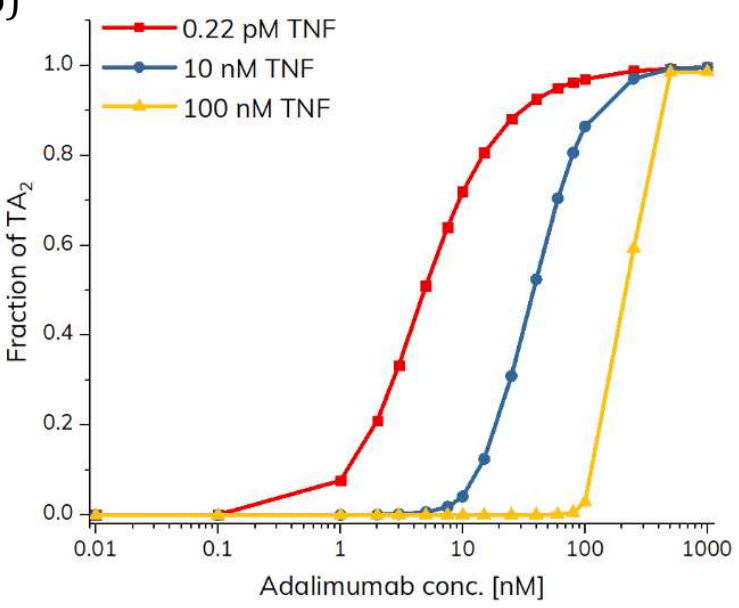

Figure 4: Simulated fractions of $(\mathrm{TA})_{2}$ (a) and $\mathrm{TA}_{2}$ (b) as a function of adalimumab concentration. Simulations were performed using TNF- $\alpha$ concentrations of $100 \mathrm{nM}, 10 \mathrm{nM}$, and $0.22 \mathrm{pM}$ (healthy physiological condition). 
Figure 4 a shows how the fraction of $(\mathrm{TA})_{2}$ is impacted by varying the TNF- $\alpha$ concentration. The $(\mathrm{TA})_{2}$ complex is dominating at intermediary adalimumab concentrations $(10$ and $100 \mathrm{nM}$, respectively) when the TNF- $\alpha$ concentration is relatively high. However, when the simulation is performed using a TNF- $\alpha$ concentration corresponding to normal blood levels $(0.22 \mathrm{pM})$, only a negligible fraction is bound in the (TA $)_{2}$ complex. In case of inflammation, the TNF- $\alpha$ level may be significantly elevated thus resulting in increased formation of $(\mathrm{TA})_{2}$. The formation of $\mathrm{TA}_{2}$ is shown at different TNF- $\alpha$ concentrations in Fig. $4 \mathrm{~b}$. As expected, the formation of this complex is shifted towards higher concentrations of adalimumab at higher concentrations of TNF- $\alpha$.

The fractions of the complexes are strongly dependent on both the relative and absolute concentrations of TNF- $\alpha$ and adalimumab. The concentrations of complex-bound and free adalimumab as well as the ability to address binding stoichiometry provides important new information on the TNF- $\alpha$ - adalimumab system. The nature and the amounts of the complexes formed is not only of importance for characterizing the mode of action on a molecular level, but it may also provide information on possible adverse effects. For instance, it is speculated that some of the formed complexes are particularly prone to immunogenic responses in the patients $20,27,28$.

The fact that the TNF- $\alpha$-adalimumab interactions results in several complexes being formed, also means that a simple $1: 1 K_{\mathrm{d}}$ does not provide a satisfactory description of the interaction and might be subject to large variations even upon small perturbations of the experimental conditions. Indeed, several techniques have been used to study the interaction between TNF- $\alpha$ and adalimumab. Krayukhina et al. used ITC $\left(K_{\mathrm{d}}=2.2 \mathrm{nM}{ }^{22}\right)$ at pH 7.4 and $25^{\circ} \mathrm{C}$. In comparison, surface based methods resulted in lower $K_{\mathrm{d}}$ values at similar $\mathrm{pH}$ and temperature: SPR $(0.10$ $\left.\mathrm{nM}^{21}, 0.18 \mathrm{nM}^{10}\right)$, BLI $\left(0.005 \mathrm{nM}^{24}\right)$, and HP-SEC $\left(0.24 \mathrm{nM}^{10}\right)$. Most of the $K_{\mathrm{d}}$ values reported in the literature assumes a 1:1 interaction, however, our results show that a strong avidity effect is needed to explain the sub-nanomolar $K_{\mathrm{d}}$ values observed with many surface binding technologies. The large variation reported in surface based $K_{d}$ values could be a result of subtle 
differences in surface coverage and orientation of immobilized TNF- $\alpha$ /adalimumab at the surface, where small differences result in a substantial impact on the observed $K_{\mathrm{d}}$, since certain experimental conditions promote strong avidity effects. For binding systems composed of several active subunits (oligomers) it is therefore advisable to use orthogonal in-solution technologies to confirm or elucidate binding mechanisms and the associated $K_{\mathrm{d}}$ values.

\section{Characterization of TNF- $\alpha$ and adalimumab interactions in buffer (capillary mixed samples)}

Characterization of biomolecular interactions using FIDA is often based on end-point measurements at equilibrium ${ }^{14,15,29}$, where binding kinetics are not probed. The experimental time frame can be precisely controlled in FIDA assays using an approach where the binding partners are mixed directly in the capillary ${ }^{30}$ (Fig. 8). This allows for an assessment of the binding kinetics. Based on the FIDA experiments, an apparent binding curve between TNF- $\alpha$ $(100 \mathrm{nM})$ and adalimumab $(0-1000 \mathrm{nM})$ was established using capillary mixing, where TNF- $\alpha-$ alexa488 and adalimumab were mixed inside the capillary during analysis, resulting in a reaction time less than 1.8 min (residence time inside the capillary). 


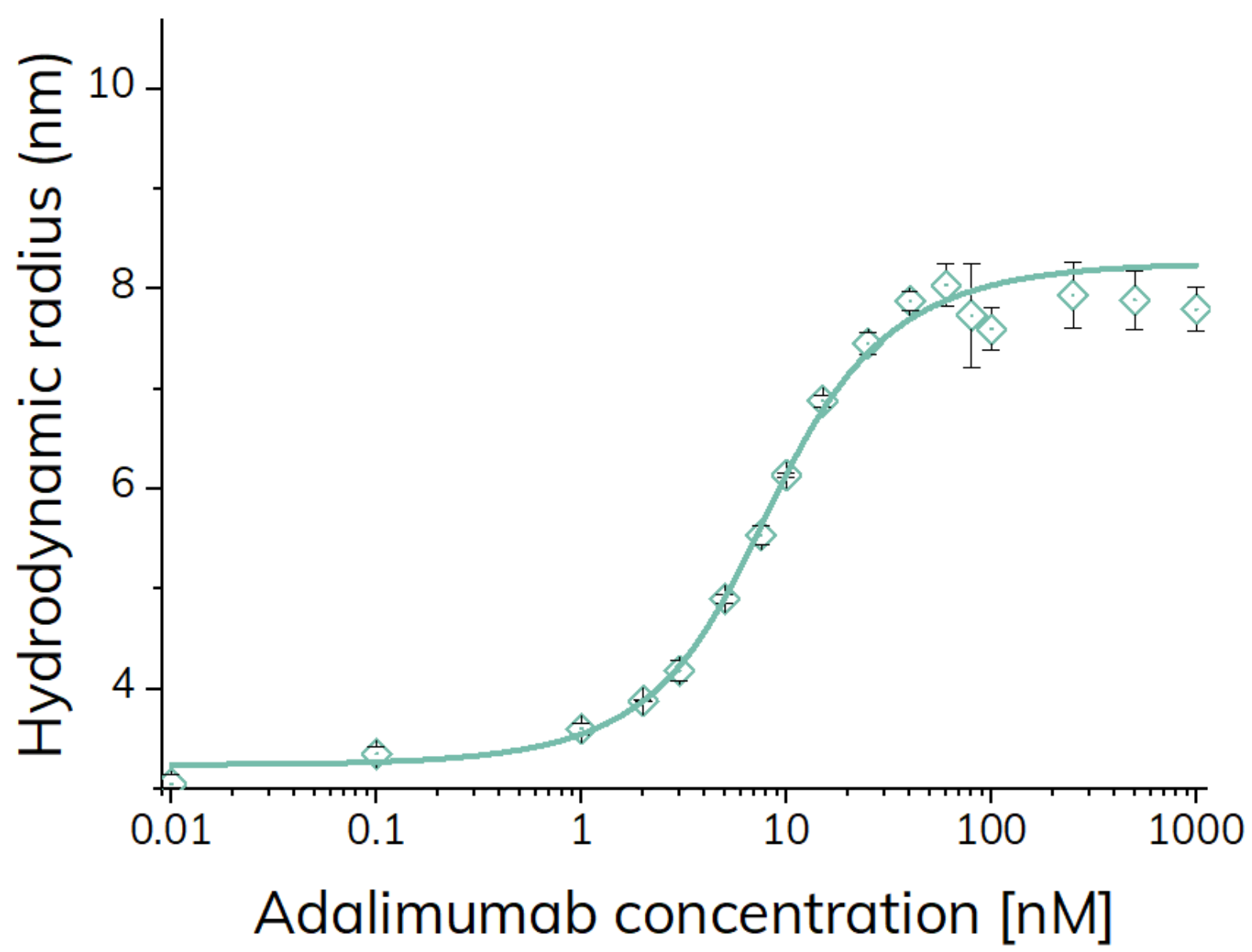

Figure 5: Apparent hydrodynamic radius of TNF- $\alpha$-alexa488 (100 $\mathrm{nM}$ ) as function of adalimumab concentration (0 - $1000 \mathrm{nM}$ ) determined by FIDA in $67 \mathrm{mM}$ phosphate buffer with $0.1 \% \mathrm{v} / \mathrm{v}$ HSA at pH 7.40 and $25^{\circ} \mathrm{C}$ ( $n=3$, error bars represent standard deviation) using capillary mixing ( $\left.<1.8 \mathrm{~min}\right)$. The solid turquoise line shows fitting to the excess indicator binding isotherm (equation 7).

The apparent size of TNF- $\alpha$ increased with increasing adalimumab concentrations and reached a plateau value around $8.0 \mathrm{~nm}$ (Fig. 5). The 1:1 excess binding model (equation 7) resulted in an apparent dissociation constant $\left(K_{\mathrm{d}}\right)$ of $1.75 \mathrm{nM}$ and complex size of $8.3 \mathrm{~nm}\left(\mathrm{R}^{2}: 0.998\right)$. These findings were analogous to the results using the pre-incubated samples (Fig. 1), except that the higher order complex (presumably $(\mathrm{TA})_{2}$ ) was not observed at equivalent concentrations (60$120 \mathrm{nM}$ in Fig. 1). Apparently, higher order complexes did not form within the experimental time frame of $1.8 \mathrm{~min}$. This suggests that the lower order complexes are formed relatively rapidly, whereas larger complexes and higher order stoichiometries require longer reaction times. The time-dependent and dynamic nature of these TNF- $\alpha$ adalimumab complexes have been observed previously in the literature using pre-incubated samples by SEC 21 . 
Characterization of TNF- $\alpha$ and adalimumab interactions in buffer (complex dissociation samples)

The dissociation kinetics of the formed TNF- $\alpha$-adalimumab complexes was probed using the socalled complex dissociation mixing approach (Fig. 8). Here, the pre-incubated samples from Fig. 1 were surrounded by neat assay buffer inside the capillary, to induce complex dissociation.

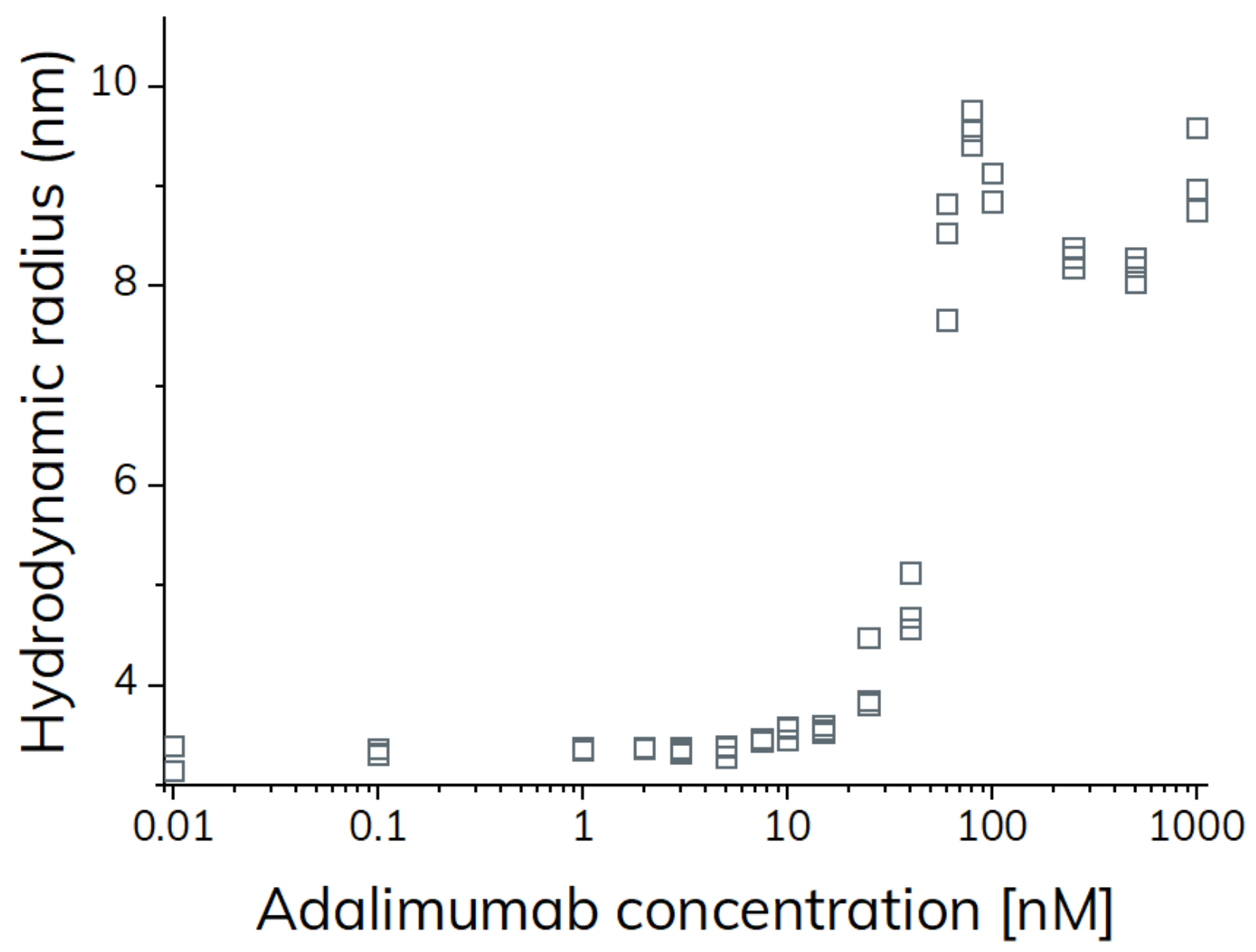

Figure 6: Apparent hydrodynamic radius of TNF- $\alpha$-alexa488 (100 nM) as function of adalimumab concentration $(0-1000 \mathrm{nM})$ determined by FIDA in $67 \mathrm{mM}$ phosphate buffer with $0.1 \% \mathrm{v} / \mathrm{v}$ HSA at pH 7.40 and $25^{\circ} \mathrm{C}$ (grey open squares) using complex dissociation.

Again, the apparent hydrodynamic radius of TNF- $\alpha$ increased with increasing adalimumab concentrations and reached a plateau value around 8.5-9.0 nm (Fig. 6). However, the apparent size change of TNF- $\alpha$ is shifted approximately an order of magnitude towards higher adalimumab concentrations as compared to Fig. 1 and 5, due to sample dilution in the capillary. The fact that no interaction is observed at low adalimumab concentration indicates a fast 
dissociation of the primary complexes ( $\mathrm{TA}, \mathrm{T}_{2} \mathrm{~A}$ and $\left.\mathrm{TA}_{2}\right)$ relative to the mobilization time inside the capillary, whereas the avidity stabilized $(\mathrm{TA})_{2}$ displays significantly slower dissociation kinetics as expected from $\mathrm{SPR}^{10}$ and hydrodynamic friction measurements 25 . According to Fig. $4 \mathrm{a}$, the maximum amount of $(\mathrm{TA})_{2}$ is observed at an adalimumab concentration around $100 \mathrm{nM}$, close to the sharp maximum at 70-80 nM adalimumab in Fig. 6. Interestingly, a second maximum is observed at the highest concentration of adalimumab (1000 nM) in Fig. 6, where the predominant species probably is the smaller $\mathrm{TA}_{2}$. Thus, the capillary dilution most likely shifts the equilibrium towards the avidity stabilized $(\mathrm{TA})_{2}$ complex. Taken together, these results highlight the importance of considering the dilution effect of the analyte (adalimumab), for systems where the binding kinetics occurs on a timescale similar to the experiment.

\section{Characterization of TNF- $\alpha$ and adalimumab interactions in human plasma}

The TNF- $\alpha$-adalimumab interactions were further characterized in human plasma since this allows for the assessment of the complex formation under physiological relevant conditions. Binding between TNF- $\alpha(100 \mathrm{nM})$ and adalimumab (0-1000 nM) was investigated in human plasma from healthy donors diluted to 10 and $20 \% \mathrm{v} / \mathrm{v}$ plasma with assay buffer. These plasma concentrations were based on the expected steady-state concentrations of adalimumab in patients receiving adalimumab treatment with 40 mg every other week ${ }^{19}$, corresponding to 5.4 and $10.8 \mathrm{nM}$ in 10 and $20 \% \mathrm{v} / \mathrm{v}$ plasma, respectively (see Supplementary Information). The dynamic range of the binding curve in Fig. 1, would therefore include relevant adalimumab plasma concentrations. The endogenous TNF- $\alpha$ concentration ${ }^{31}$ is several orders of magnitude below the indicator concentration of $100 \mathrm{nM}$, and thus not expected to interfere with the FIDA assay (see Supplementary Information).

The hydrodynamic radius of TNF- $\alpha$, in absence of adalimumab ( $0 \mathrm{nM})$, was determined to be 3.3 $\pm 0.16 \mathrm{~nm}$ and $3.6 \pm 0.32 \mathrm{~nm}$ in 10 and $20 \% \mathrm{v} / \mathrm{v}$ plasma, respectively, implying only very limited binding of TNF- $\alpha$ to plasma components when comparing with $3.20 \pm 0.04 \mathrm{~nm}$ in neat assay buffer. 
The binding curves (Fig. 7) showed an apparent increase in the size of TNF- $\alpha$ with increasing adalimumab concentrations as well as hook-shaped curves, similarly to the binding curve obtained in assay buffer (Fig. 1).

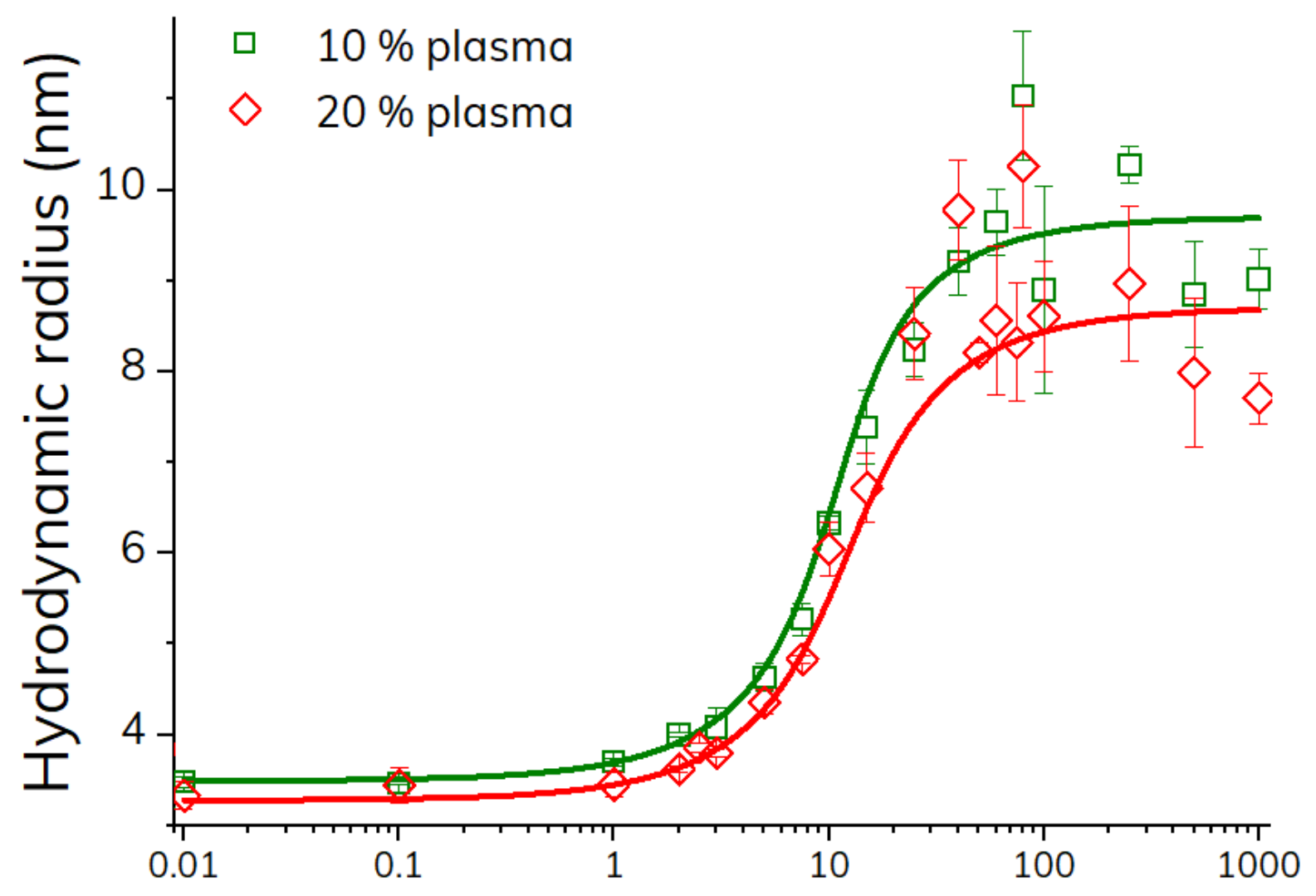

\section{Adalimumab concentration [nM]}

Figure 7: Apparent hydrodynamic radius of TNF- $\alpha$-alexa488 (100 nM) as function of adalimumab concentration $(0-1000 \mathrm{nM})$ in $10 \% \mathrm{v} / \mathrm{v}$ human plasma (green squares) and $20 \% \mathrm{v} / \mathrm{v}$ human plasma (red diamonds), determined by FIDA at $25^{\circ} \mathrm{C}(n=3$, error bars represent standard deviation) using preincubated samples (> $10 \mathrm{~min}$ ). The solid lines represent fitting to the excess indicator binding isotherm (equation 7) with $R^{2}$ of 0.987 (green) and 0.979 (red), respectively.

The 1:1 excess binding model (equation 7) resulted in apparent $K_{\mathrm{d}}$ values of 1.01 and $1.73 \mathrm{nM}$, and complex sizes of 9.7 and $8.7 \mathrm{~nm}$ in 10 and $20 \% \mathrm{v} / \mathrm{v}$ plasma, respectively. Overall, these findings are in line with the apparent values in assay buffer $\left(K_{\mathrm{d}}: 1.52 \mathrm{nM}\right.$, complex size: $\left.8.9 \mathrm{~nm}\right)$. The minor differences observed in complex size as function of adalimumab concentration emphasize that attention must be drawn to performing interaction and characterization assays under physiological relevant conditions, as they might differ from experiments performed in simple buffer system. 


\section{Conclusions}

The present work demonstrates the application of FIDA assays for size-based and in-solution characterization of the interaction between TNF- $\alpha$ and adalimumab in both phosphate buffer and human plasma. We selectively measured the hydrodynamic radius of TNF- $\alpha$ in a titration series experiment with adalimumab, thereby determining the dissociation constant $\left(K_{\mathrm{d}}\right)$ and complex sizes as well as confirming the oligomeric state of TNF- $\alpha$. We found that the interaction involved generation of higher order complexes and substantial avidity effects. Therefore, the binding mechanism was modelled and simulated, demonstrating that the formation of higher order complexes highly depends on absolute and relative TNF- $\alpha$ and adalimumab concentrations. Furthermore, we performed an assessment of the binding kinetics, showing that smaller and lower order stoichiometries were rapidly formed whereas larger and higher order complexes required longer reaction time to form. Finally, the TNF- $\alpha$-adalimumab interaction was measured in human plasma under physiological relevant conditions, implying similarities to the buffered model system, but also slight differences, thus stressing the importance of measuring in biological samples. The developed FIDA assays are applicable to measurement of both binding affinity and structural changes of biomolecular interactions under biorelevant conditions in a single assay format. 


\section{Experimental}

\section{Materials and chemicals}

Recombinant human TNF- $\alpha$ (cat. no. ab155699) was procured from Abcam (Cambridge, United Kingdom). Adalimumab from Amgen Europe B.V. (Amgevita ${ }^{\circledR}$, Breda, Netherlands) was bought from Nomeco (Copenhagen, Denmark). Plasma samples from healthy donors were provided by Rigshospitalets Blodbank (Copenhagen, Denmark). Alexa Fluor 488 antibody labeling kit (cat. no. A20181) was purchased from Thermo Fisher (Waltham, MA, USA). Human serum albumin (HSA) was purchased from Sigma-Aldrich (St. Louis, Missouri, USA). Disodium hydrogen phosphate dihydrate, and sodium dihydrogen phosphate monohydrate were purchased from Merck (Darmstadt, Germany). Purified water $\left(18.2 \mathrm{M} \Omega\right.$-cm at $\left.25^{\circ} \mathrm{C}\right)$ was obtained from an SG Ultraclear water purification system (SG Water, Barsbuttel, Germany).

\section{Assay buffer}

Phosphate buffer (pH 7.4; $67 \mathrm{mM}$ ) was prepared with purified water and filtered using a Q-max $0.45 \mu \mathrm{m}$ nylon syringe filter (Frisenette, Knebel, Denmark) prior to use.

\section{Fluorophore labelling of TNF- $\alpha$}

TNF- $\alpha$ was labelled with the Alexa Fluor 488 antibody labeling kit via tetrafluorophenyl (TFP) ester coupling chemistry, and subsequently purified, following the supplier's instructions ${ }^{32}$. The TNF- $\alpha$-alexa488 conjugate concentration was determined using a microvolume spectrophotometer (NanoDrop One, Thermo Fisher Scientific, Wilmington, Delaware, USA) applying the extinction coefficient of TNF- $\alpha\left(\varepsilon=60500 \mathrm{~cm}^{-1} \mathrm{M}^{-1}\right)$ at $280 \mathrm{~nm}^{33}$ assuming trimeric TNF- $\alpha$ in phosphate buffer (pH 7.4; $67 \mathrm{mM})$.

\section{Equipment}

FIDA experiments were conducted using a FIDA 1 instrument employing light-emitting-diode (LED) induced fluorescence detection with an excitation wavelength of $480 \mathrm{~nm}$ and emission 
wavelength > $515 \mathrm{~nm}$ (Fida Biosystems ApS, Copenhagen, Denmark). TDA experiments were performed using a FIDA 1 instrument coupled to an intrinsic laser-induced-fluorescence (LIF) detector (ZETALIF evolution) with an excitation wavelength of $266 \mathrm{~nm}$ and emission wavelength of 300 - $760 \mathrm{~nm}$ (Picometrics, Labege, France).

PEG-coated capillaries with inner diameter $75 \mu \mathrm{m}$, outer diameter $375 \mu \mathrm{m}$, total length $100 \mathrm{~cm}$, and length to detection window $84 \mathrm{~cm}$ (Fida Biosystems) were used for all experiments.

\section{Methods}

\section{FIDA and TDA procedures}

For the TDA and FIDA experiments, the following protocol was applied. First, assay buffer (pH 7.4; $67 \mathrm{mM}$ ) was used for flushing and equilibrating the PEG-coated capillary at 1500 mbar for $300 \mathrm{~s}$. Subsequently, the analyte solution was filled into the capillary at 1500 mbar for $45 \mathrm{~s}$ followed by the indicator sample at 50 mbar for $10 \mathrm{~s}$. Finally, the indicator sample was mobilized to the fluorescence detector with analyte solution at 400 mbar for $180 \mathrm{~s}$. The final mobilization step of the FIDA experiments conducted in plasma was set to $240 \mathrm{~s}$ at 400 mbar due to an increase in sample viscosity.

\section{Hydrodynamic radius of adalimumab}

The hydrodynamic radius of adalimumab $(1 \mathrm{mg} / \mathrm{mL})$ was measured in assay buffer by TDA employing intrinsic fluorescence detection.

\section{Binding curve in buffer}

Binding curves were generated using FIDA in assay buffer containing human serum albumin $(0.1 \%$ $\mathrm{v} / \mathrm{v}$ ) as surface passivation agent. The TNF- $\alpha$-alexa488 (indicator) concentration was fixed to 100 $\mathrm{nM}$ and titrated with adalimumab (analyte) in the concentration range $0-1000 \mathrm{nM}$. Three different incubation and mixing modes were used (see Fig. 8): 1) Pre-incubation where TNF- $\alpha$ alexa488 and adalimumab were pre-incubated ( $>10 \mathrm{~min}$ ) prior to the FIDA measurements, and subsequently analyzed with adjacent zones of equal adalimumab concentration; 2) Capillary mix 
where TNF- $\alpha$-alexa488 was mixed with adalimumab inside the capillary during the mobilization period ( 2-3 min); 3) Complex dissociation where TNF- $\alpha$-alexa488 and adalimumab were preincubated ( $>10 \mathrm{~min}$ ) prior to the FIDA measurements, and then analyzed with adjacent zones of neat assay buffer.

\section{Modes}

1) Pre-incubation

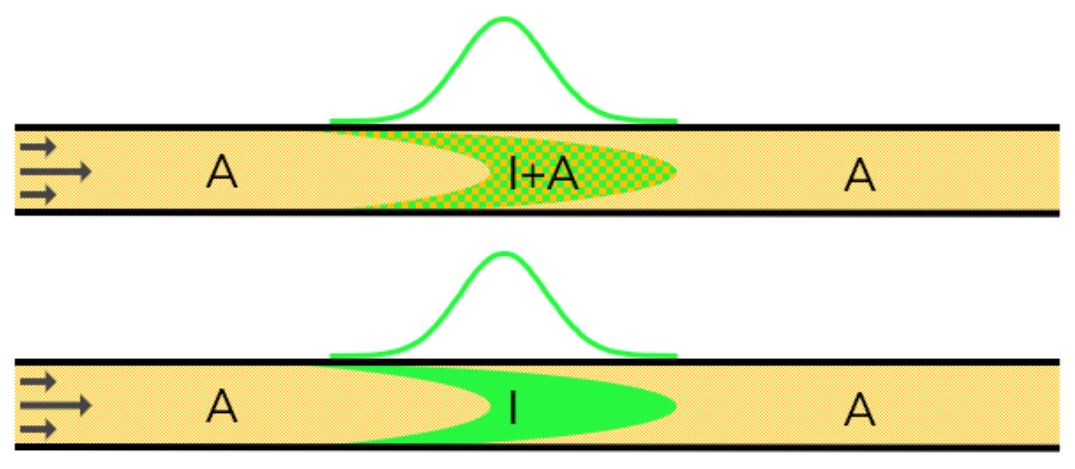

2) Capillary mix

$\stackrel{\vec{\rightrightarrows}}{\rightrightarrows} \mathrm{A}-\mathrm{A}$

3) Complex dissociation

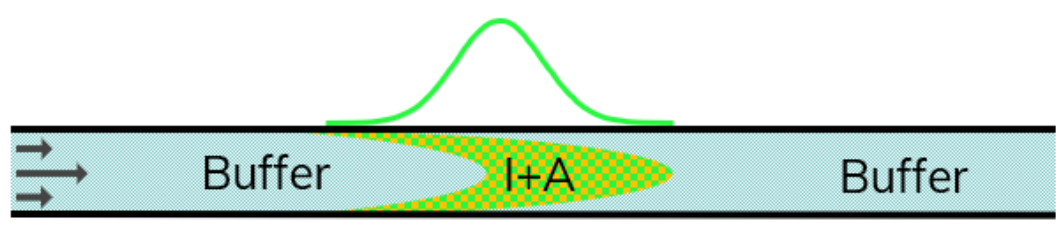

Figure 8: Schematic showing the applied incubation and mixing modes in the FIDA experiments, allowing characterization of in-solution binding kinetics.

\section{Binding curves in plasma}

Binding curves were generated using FIDA in 10 and $20 \% \mathrm{v} / \mathrm{v}$ human plasma diluted with assay buffer. The TNF- $\alpha$-alexa488 concentration was fixed to $100 \mathrm{nM}$ and titrated with adalimumab in the concentration range $0-1000 \mathrm{nM}$. TNF- $\alpha$-alexa488 was pre-incubated with adalimumab in the sample vials for $>10$ min to attain equilibrium before the FIDA measurements.

\section{Data analysis}

The Taylorgrams were processed using the FIDA software suite, version 1.1 (Fida Biosystems ApS, Copenhagen, Denmark), in order to calculate the apparent hydrodynamic radius of TNF- $\alpha$ alexa488 (indicator) at each adalimumab concentration (analyte). The Taylorgram fraction 
setting was set to $75 \%$ for all data points, and changes in sample viscosity were corrected according to a reference measurement at $25^{\circ} \mathrm{C}$, as previously described 14 .

A binding isotherm, assuming 1:1 binding and excess of indicator, was used for fitting the obtained FIDA measurements, i.e., apparent hydrodynamic radius of TNF- $\alpha$-alexa488 as function of adalimumab concentration using the FIDA software ${ }^{17}$ :

$R_{\mathrm{app}}=\left(\begin{array}{l}\left(R_{\mathrm{IA}}\right)^{-1} \cdot\left(\frac{\left.\left(C_{I}+C_{A}+K_{d}\right)-\sqrt{\left(C_{I}+C_{A}+K_{d}\right)^{2}-4 \cdot C_{A} \cdot C_{I}}\right)}{2 \cdot C_{I}}\right) \\ +\left(R_{\mathrm{I}}\right)^{-1} \cdot\left(\frac{\left.\left(C_{I}-C_{A}-K_{d}\right)+\sqrt{\left(C_{I}+C_{A}+K_{d}\right)^{2}-4 \cdot C_{A} \cdot C_{I}}\right)}{2 \cdot C_{I}}\right)\end{array}\right)^{-1}$

where $R_{\mathrm{app}}, R_{\mathrm{J}}$ and $R_{\mathrm{IA}}$ are the apparent, indicator and complex hydrodynamic radii, respectively, $K_{\mathrm{d}}$ is the dissociation constant, and $C_{\mathrm{I}}$ and $C_{\mathrm{A}}$ are the formal concentrations of the indicator and analyte, respectively.

An extended binding model for multiple and higher order stoichiometries is derived in the results and discussion paragraph as well as in the Supplementary Information.

\section{Data availability}

The datasets generated and analysed during the current study are available from the corresponding author on request. 


\section{References}

1. Kenakin, T. P. Pharmacology in drug discovery and development: Understanding drug response: Second edition. Pharmacology in Drug Discovery and Development: Understanding Drug Response: Second Edition (2016).

2. Terstappen, G. C., Schlüpen, C., Raggiaschi, R. \& Gaviraghi, G. Target deconvolution strategies in drug discovery. Nat. Rev. Drug Discov. 6, 891-903 (2007).

3. Zhang, R. \& Monsma, F. Binding kinetics and mechanism of action: Toward the discovery and development of better and best in class drugs. Expert Opin. Drug Discov. 5, 10231029 (2010).

4. ICH Expert Working Group. ICH Guideline Q6B. Specifications: Test Procedures and Acceptance Critreia for Biotechnological/Biological Products (1999).

5. US Food and Drug administration (Kathryn C. Zoon). Points to consider in the manufacture and testing of monoclonal antibody products for human use (1997). 50 (1997). doi:10.1097/00002371-199705000-00007

6. Robinson, C. J. \& Jones, C. Quality control and analytical techniques for biopharmaceuticals. Bioanalysis 3, 81-95 (2011).

7. WHO. Guidelines on the quality, safety and efficacy of biotherapeutic protein products prepared by recombinant DNA technology. Who 91 (2013).

8. Liang, Z., Glick, J. \& Flarakos, J. Bioanalytical Challenges in Support of Complex Modalities of Antibody-Based Therapeutics. AAPS J. 22, 1-6 (2020).

9. Lim, H. et al. Structural biology of the TNF- $\alpha$ antagonists used in the treatment of rheumatoid arthritis. Int. J. Mol. Sci. 19, (2018).

10. Van Schie, K. A. et al. Therapeutic TNF Inhibitors can Differentially Stabilize Trimeric TNF by Inhibiting Monomer Exchange. Sci. Rep. 6, (2016).

11. Corti, A., Fassina, G., Marcucci, F., Barbanti, E. \& Cassani, G. Oligomeric tumour necrosis factor $\alpha$ slowly converts into inactive forms at bioactive levels. Biochem. J. 284, 905-910 (1992).

12. Filipe, V., Hawe, A., Carpenter, J. F. \& Jiskoot, W. Analytical approaches to assess the degradation of therapeutic proteins. TrAC - Trends in Analytical Chemistry 49, 118-125 (2013).

13. Jensen, H. \& Østergaard, J. Flow induced dispersion analysis quantifies noncovalent interactions in nanoliter samples. J. Am. Chem. Soc. 132, 4070-4071 (2010).

14. Pedersen, M. E., Gad, S. I., Østergaard, J. \& Jensen, H. Protein Characterization in 3D: Size, Folding, and Functional Assessment in a Unified Approach. Anal. Chem. 91, 4975-4979 (2019).

15. Poulsen, N. N. et al. Flow-Induced Dispersion Analysis for Probing Anti-dsDNA Antibody Binding Heterogeneity in Systemic Lupus Erythematosus Patients: Toward a New Approach for Diagnosis and Patient Stratification. Anal. Chem. 88, 9056-9061 (2016).

16. Cholak, E.; Bugge, K.; Khondker, A.; Gauger, K.; Pedraz-Cuesta, E.; Pedersen, M. E.; Bucciarelli, S.; Vestergaard, B.; Pedersen, S. F.; Rheinstädter, M. C.; Langkilde, A. E.; Kragelund, B. B. Avidity within the N-terminal anchor drives $\alpha$-synuclein membrane 
interaction and insertion. FASEB J. 0, 1-21 (2020).

17. Pedersen, M. E., Østergaard, J. \& Jensen, H. In-Solution IgG Titer Determination in Fermentation Broth Using Affibodies and Flow-Induced Dispersion Analysis. ACS Omega 5, 10519-10524 (2020).

18. Poulsen, N. N. et al. Flow induced dispersion analysis rapidly quantifies proteins in human plasma samples. Analyst 140, 4365-4369 (2015).

19. Abbvie. Humira Product monograph. 174 (2019).

20. Kohno, T., Tam, L. T. T., Stevens, S. R. \& Louie, J. S. Binding characteristics of tumor necrosis factor receptor-Fc fusion proteins vs anti-tumor necrosis factor mAbs. J. Investig. Dermatology Symp. Proc. 12, 5-8 (2007).

21. Santora, L. C., Kaymakcalan, Z., Sakorafas, P., Krull, I. S. \& Grant, K. Characterization of noncovalent complexes of recombinant human monoclonal antibody and antigen using cation exchange, size exclusion chromatography, and BIAcore. Anal. Biochem. 299, 119129 (2001).

22. Krayukhina, E. et al. Analytical ultracentrifugation with fluorescence detection system reveals differences in complex formation between recombinant human TNF and different biological TNF antagonists in various environments. MAbs 9, 664-679 (2017).

23. Tran, B. N. et al. Higher order structures of Adalimumab, Infliximab and their complexes with TNFa revealed by electron microscopy. Protein Sci. 26, 2392-2398 (2017).

24. Schröter, C. et al. A generic approach to engineer antibody $\mathrm{pH}$-switches using combinatorial histidine scanning libraries and yeast display. MAbs 7, 138-151 (2015).

25. Daub, H. et al. The trimer to monomer transition of Tumor Necrosis Factor-Alpha is a dynamic process that is significantly altered by therapeutic antibodies. Sci. Rep. 10, (2020).

26. Hawe, A., Hulse, W. L., Jiskoot, W. \& Forbes, R. T. Taylor dispersion analysis compared to dynamic light scattering for the size analysis of therapeutic peptides and proteins and their aggregates. Pharm. Res. 28, 2302-2310 (2011).

27. Jefferis, R. Aggregation, immune complexes and immunogenicity. MAbs 3, 503-504 (2011).

28. Van Schouwenburg, P. A., Rispens, T. \& Wolbink, G. J. Immunogenicity of anti-TNF biologic therapies for rheumatoid arthritis. Nat. Rev. Rheumatol. 9, 164-172 (2013).

29. Restan, M. S., Pedersen, M. E., Jensen, H. \& Pedersen-Bjergaard, S. Electromembrane Extraction of Unconjugated Fluorescein Isothiocyanate from Solutions of Labeled Proteins Prior to Flow Induced Dispersion Analysis. Anal. Chem. (2019). doi:10.1021/acs.analchem.9b00730

30. Pedersen, M. E., Østergaard, J. \& Jensen, H. Flow-induced dispersion analysis (FIDA) for protein quantification and characterization. in Methods in Molecular Biology (2019). doi:10.1007/978-1-4939-9213-3_8

31. Arican, O., Aral, M., Sasmaz, S. \& Ciragil, P. Serum levels of TNF- $\alpha$, IFN- $\gamma$, IL-6, IL-8, IL-12, IL-17, and IL-18 in patients with active psoriasis and correlation with disease severity. Mediators Inflamm. 5, 273-279 (2005). 
32. Life Technologies. Alexa Fluor ${ }^{\circledR}$, Pacific Blue ${ }^{\mathrm{TM}}$, and Pacific Orange ${ }^{\mathrm{TM}}$ Antibody Labeling Kits. 9 (2012).

33. Marušič, J. et al. Recognition of human tumor necrosis factor $\alpha$ (TNF- $\alpha$ ) by therapeutic antibody fragment: Energetics and structural features. J. Biol. Chem. 287, 8613-8620 (2012). 


\section{Author contributions}

Study hypothesis and design: MEP and HJ.

Deriving higher order model: $\mathrm{HJ}$.

Performing FIDA experiments: RMSH and MEP.

Data analysis: RMSH and MEP.

Interpretation of results: MEP, RMSH, HJ, and JØ.

Manuscript preparation: MEP, HJ, JØ and RMSH.

All authors have reviewed and approved the final manuscript.

\section{Acknowledgements}

This work was supported by the Innovation Fund Denmark (grant number 9065-00009B).

\section{Competing Interests}

The authors declare the following competing financial interests: HJ, JØ, and MEP have commercial interest in Fida Biosystems ApS. MEP and HJ are employees of Fida Biosystems ApS. RMSH has no competing interests.

\section{Supplementary information}

Supplementary Information is available online. 
Figures

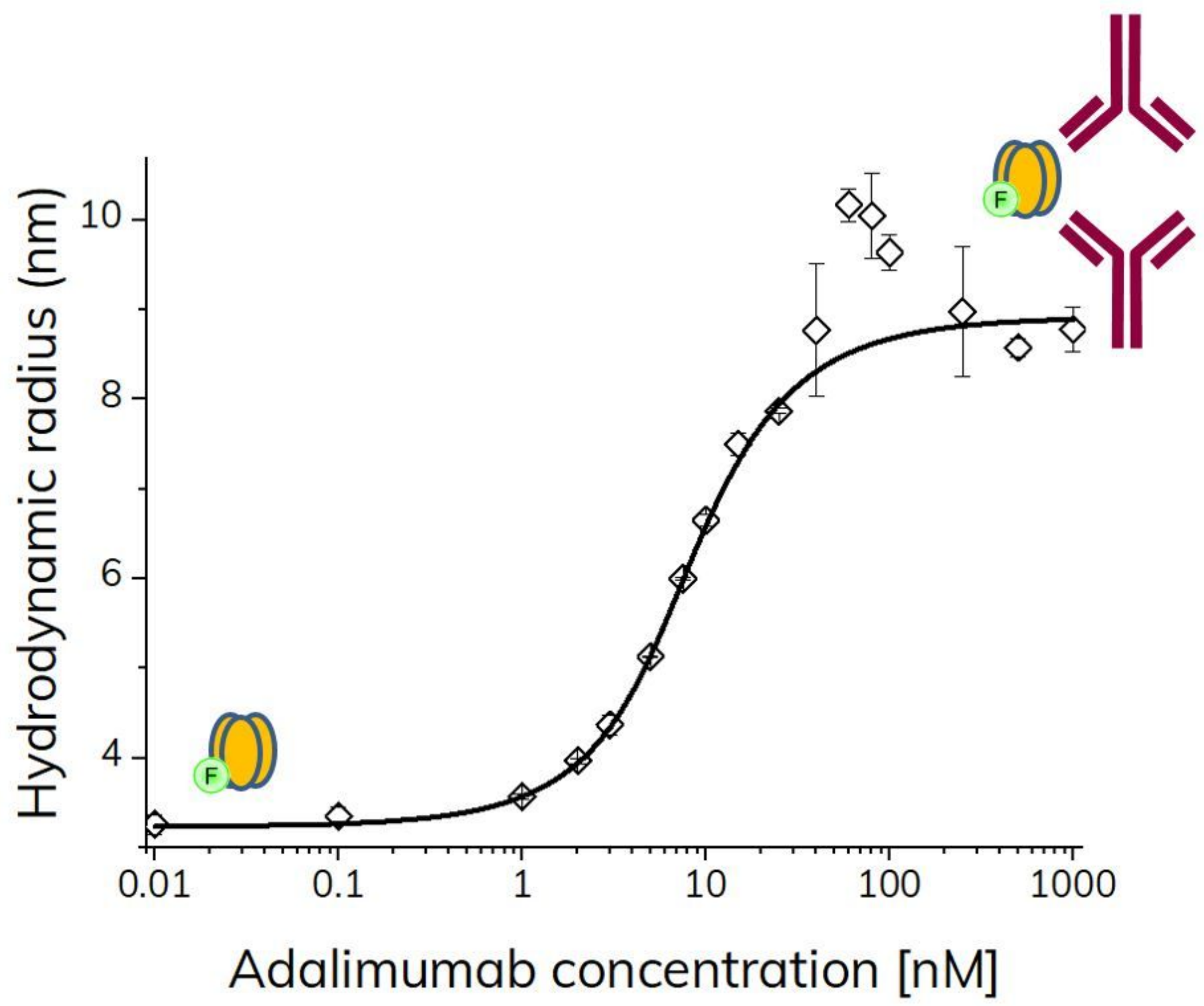

Figure 1

Apparent hydrodynamic radius of TNF-a-alexa488 (100 nM) as function of adalimumab concentration ( 0 - $1000 \mathrm{nM}$ ) determined by FIDA in $67 \mathrm{mM}$ phosphate buffer with $0.1 \% \mathrm{v} / \mathrm{v} \mathrm{HSA}$ at pH 7.40 and $25^{\circ} \mathrm{C}$ using pre-incubated samples ( $n=3$, error bars represent standard deviation). The solid line shows fitting to the excess indicator binding isotherm (equation 7$)$. 
a)

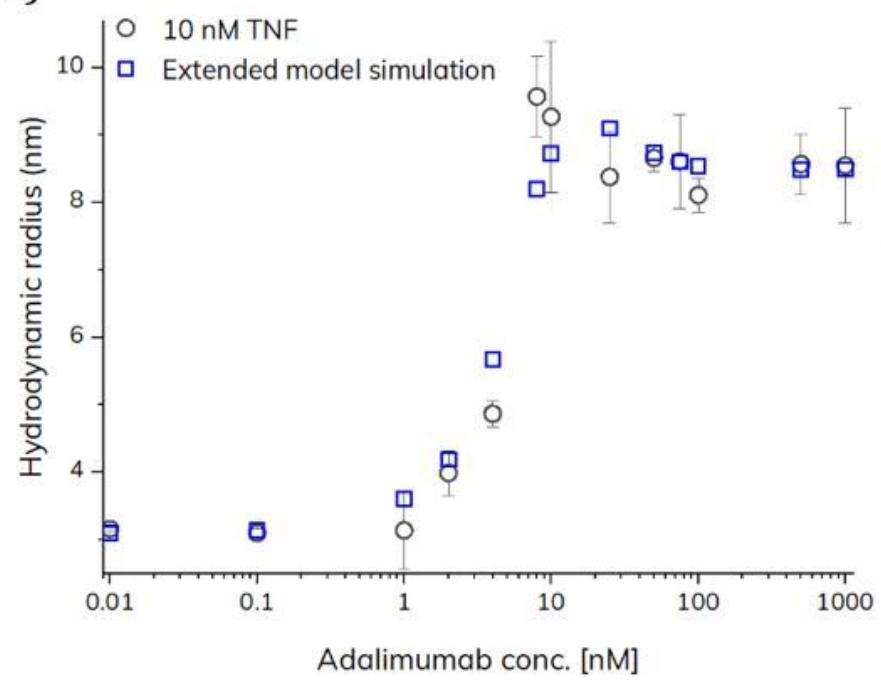

b)

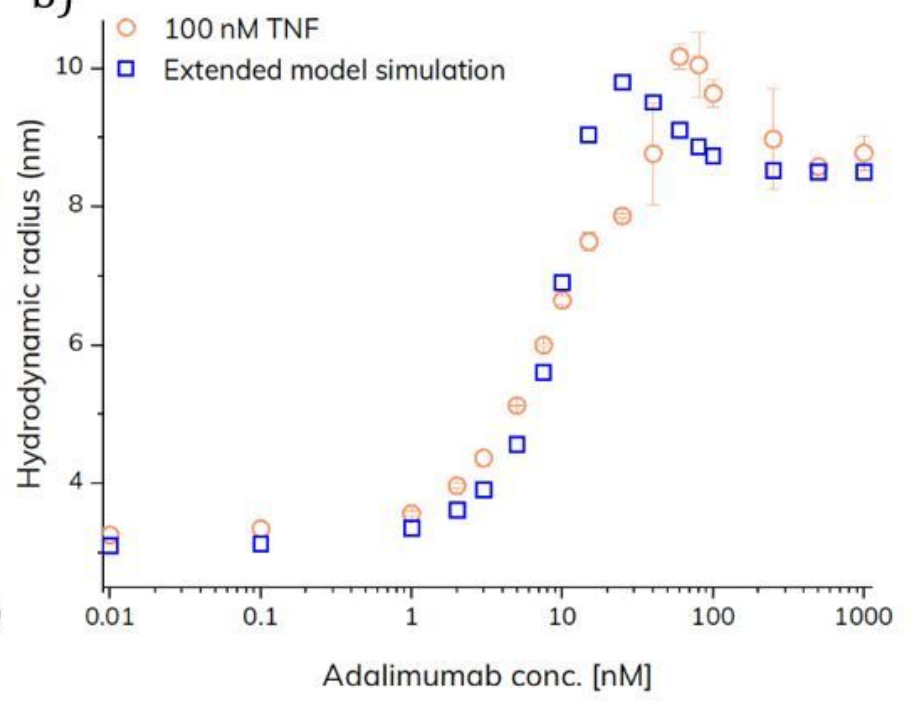

Figure 2

Extended model simulations of 10 (a) and 100 (b) nM TNF-a-alexa488 (open squares) as function of adalimumab concentration $(0-1000 \mathrm{nM})$ compared to FIDA measurements in $67 \mathrm{mM}$ phosphate buffer with $0.1 \% \mathrm{v} / \mathrm{v} \mathrm{HSA}$ at pH 7.40 and $25^{\circ} \mathrm{C}$ using pre-incubated samples (open circles, $\mathrm{n}=3$, error bars represent standard deviation). 


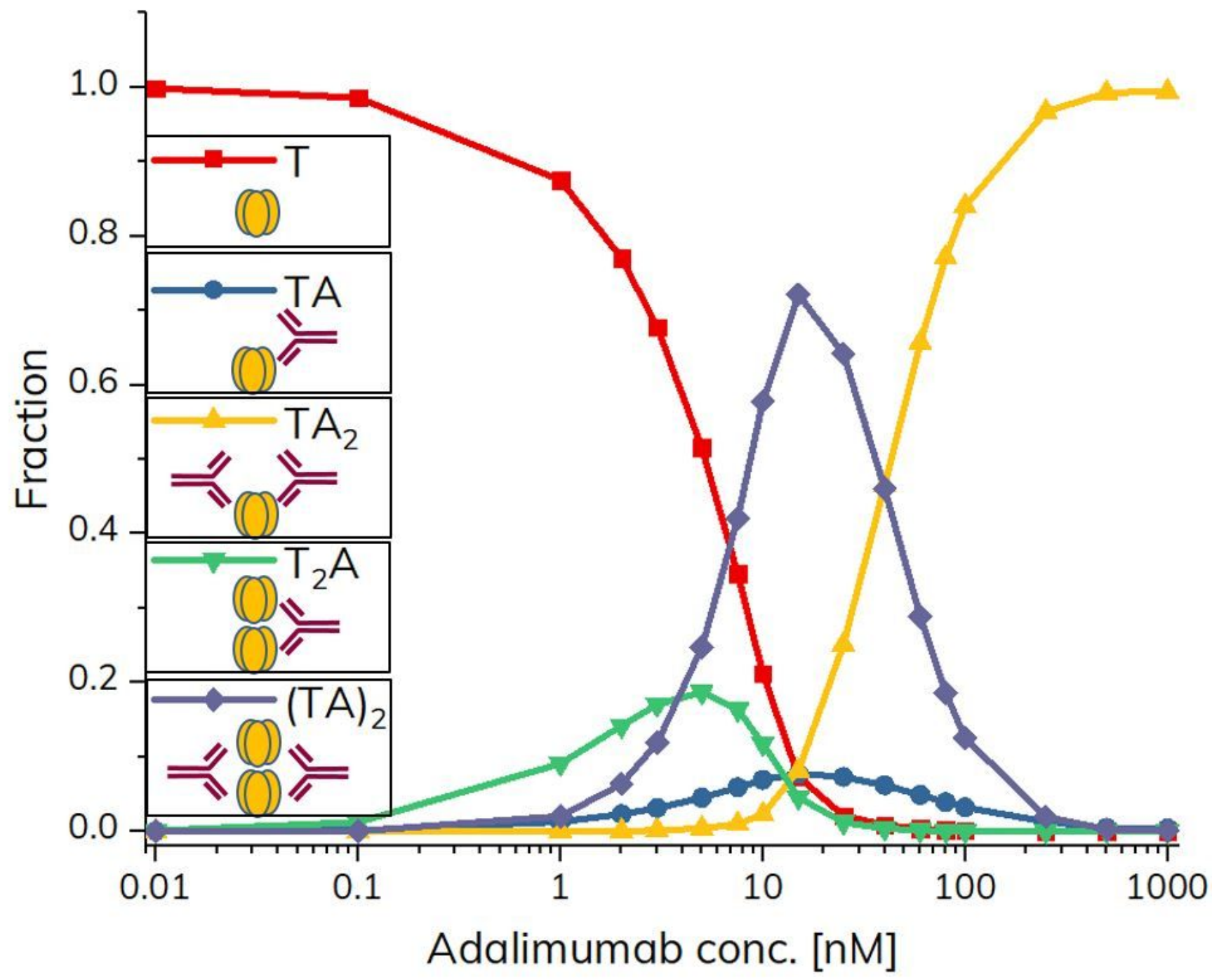

Figure 3

Simulated fractions of the various complexes between TNF- $a$ and adalimumab as a function of adalimumab concentration corresponding to the experimental dataset at $100 \mathrm{nM}$ TNF-a in the preincubated samples. Data points are based on the extended model simulations. 
a)

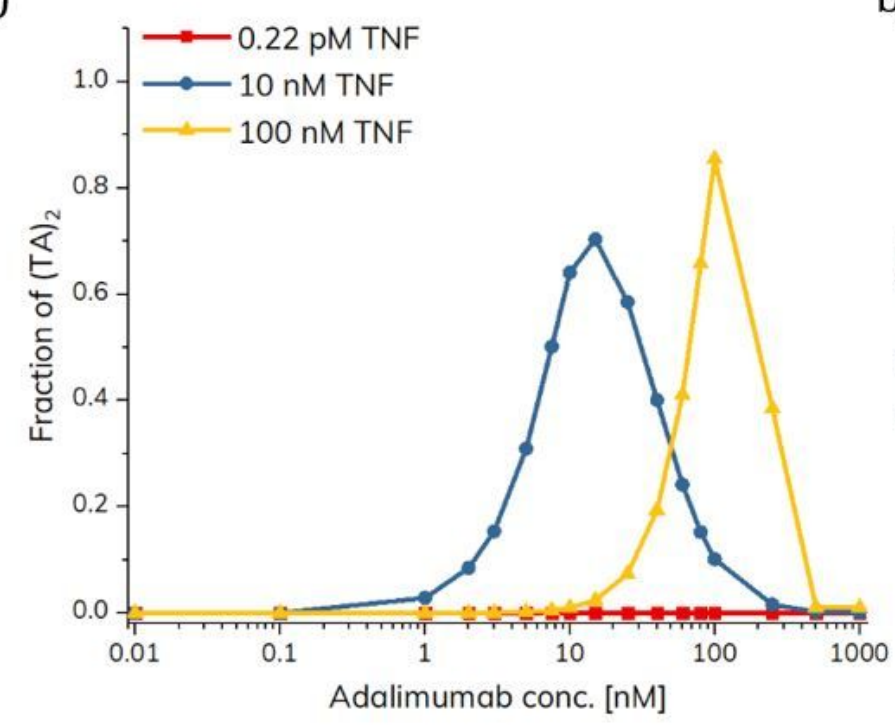

b)

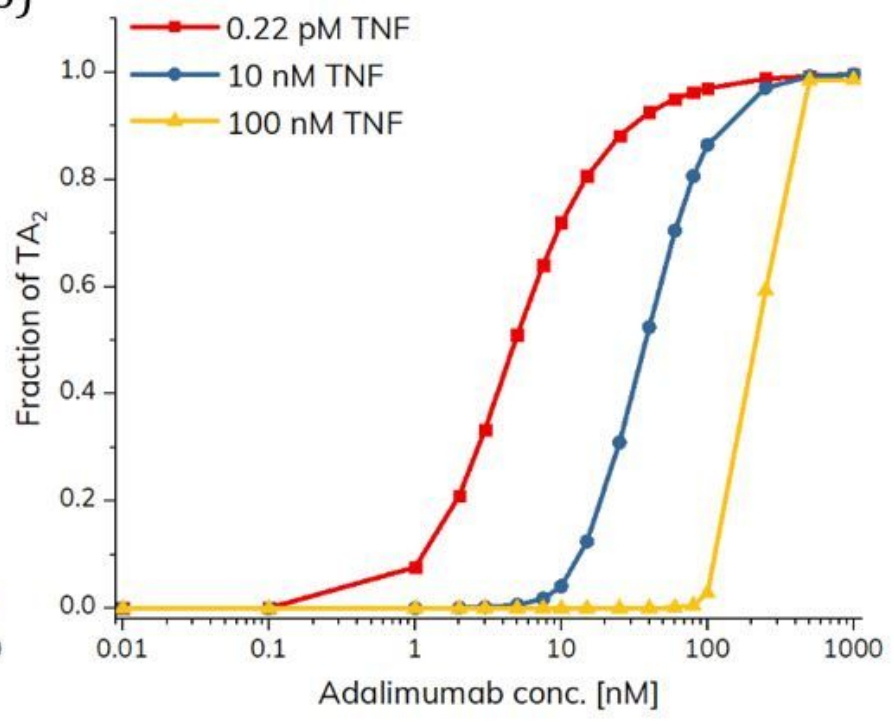

\section{Figure 4}

Simulated fractions of (TA)2 (a) and TA2 (b) as a function of adalimumab concentration. Simulations were performed using TNF- $a$ concentrations of $100 \mathrm{nM}, 10 \mathrm{nM}$, and $0.22 \mathrm{pM}$ (healthy physiological condition). 


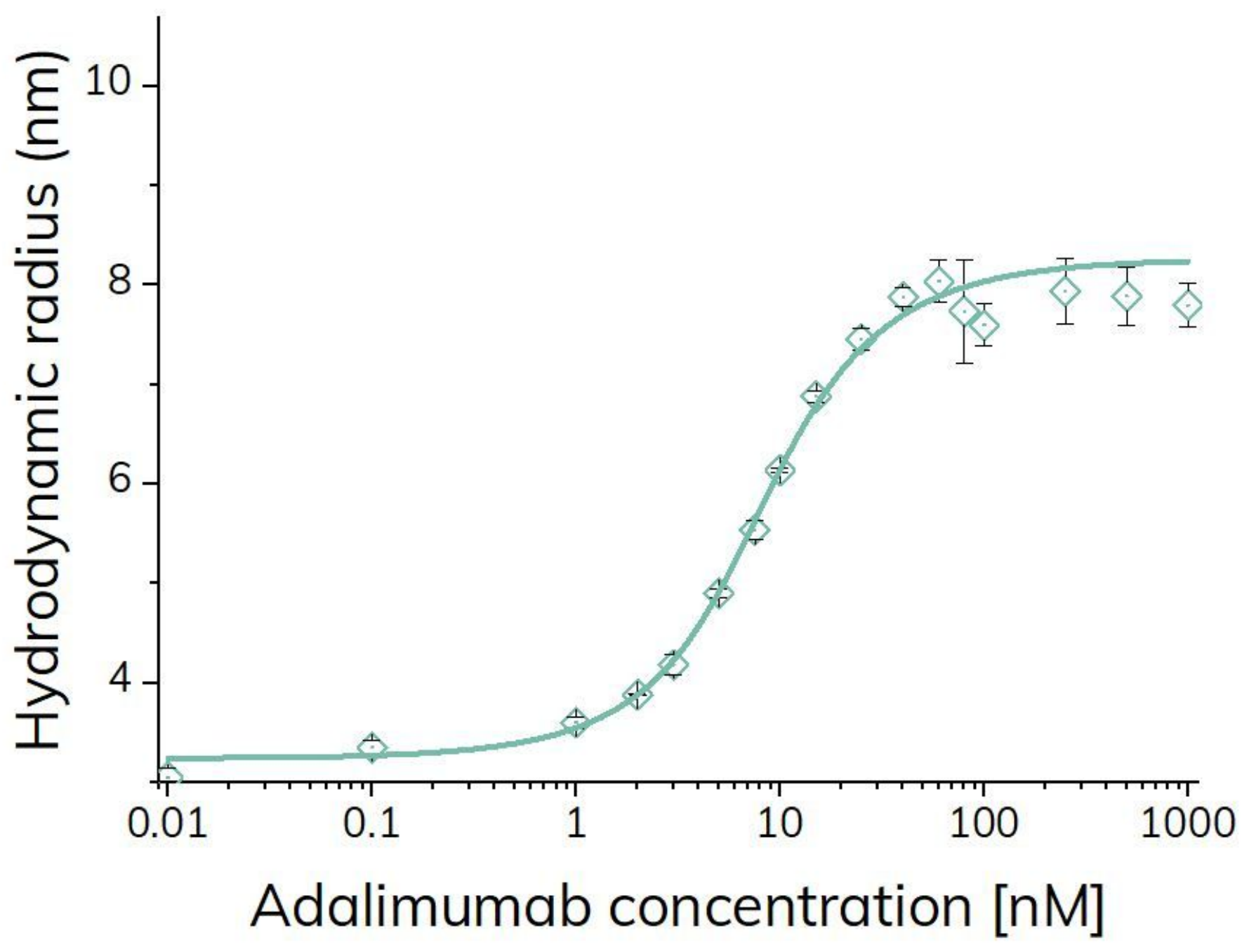

Figure 5

Apparent hydrodynamic radius of TNF-a-alexa488 $(100 \mathrm{nM})$ as function of adalimumab concentration (0 - $1000 \mathrm{nM})$ determined by FIDA in $67 \mathrm{mM}$ phosphate buffer with $0.1 \% \mathrm{v} / \mathrm{v} \mathrm{HSA}$ at pH 7.40 and $25^{\circ} \mathrm{C}(\mathrm{n}=$ 3 , error bars represent standard deviation) using capillary mixing $(<1.8 \mathrm{~min})$. The solid turquoise line shows fitting to the excess indicator binding isotherm (equation 7 ). 


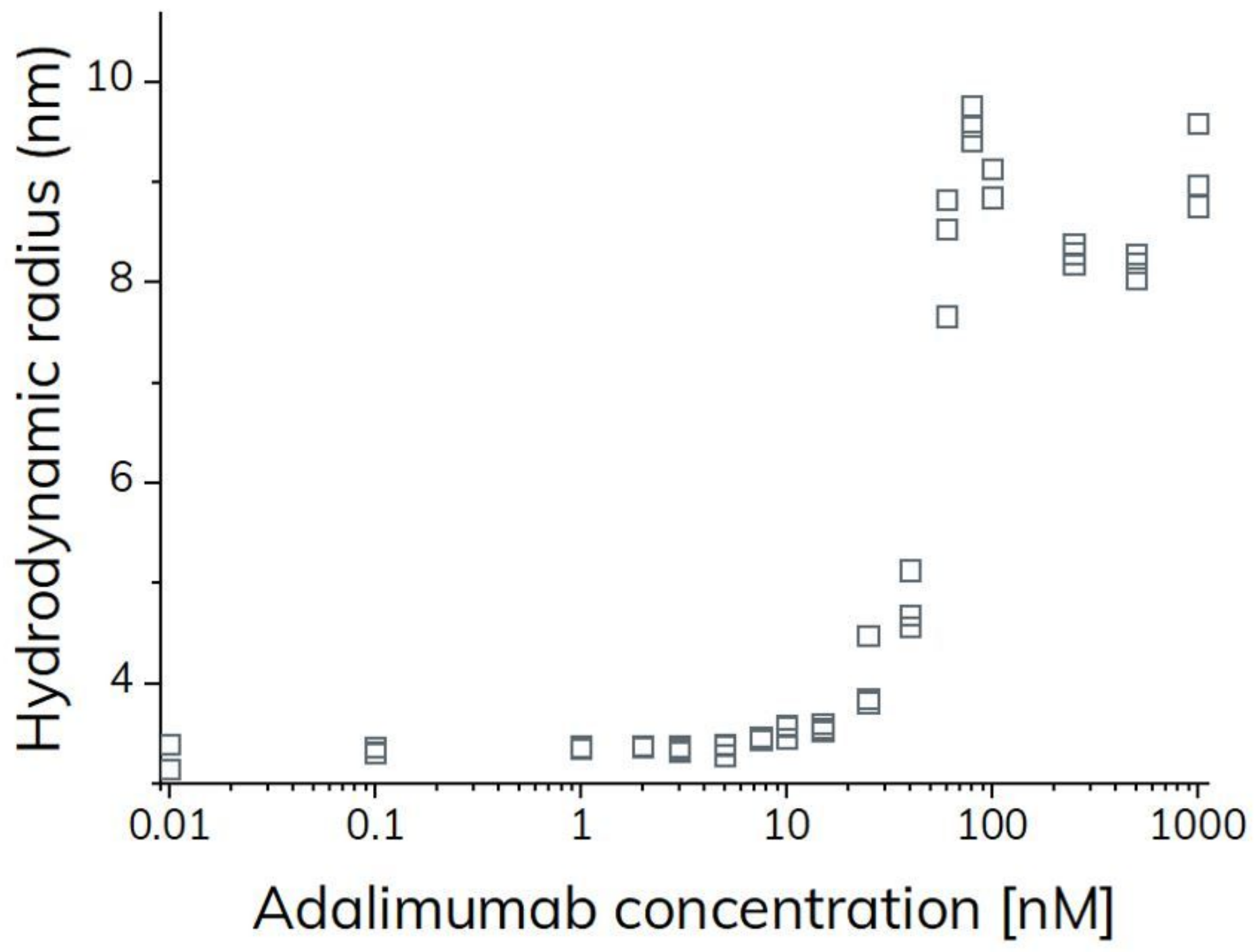

Figure 6

Apparent hydrodynamic radius of TNF-a-alexa488 $(100 \mathrm{nM})$ as function of adalimumab concentration ( 0 - $1000 \mathrm{nM}$ ) determined by FIDA in $67 \mathrm{mM}$ phosphate buffer with $0.1 \% \mathrm{v} / \mathrm{v} \mathrm{HSA}$ at pH 7.40 and $25^{\circ} \mathrm{C}$ (grey open squares) using complex dissociation. 


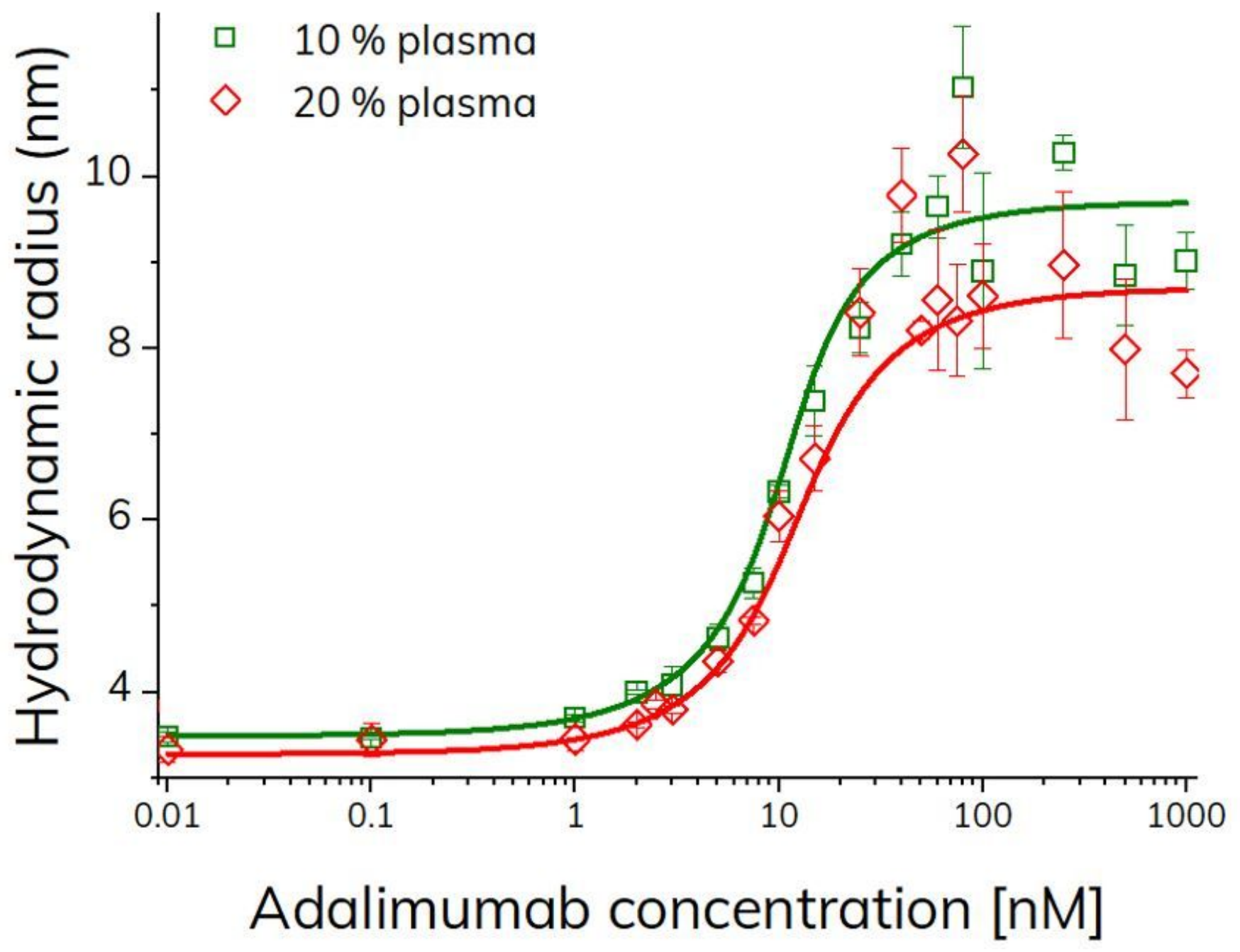

Figure 7

Apparent hydrodynamic radius of TNF-a-alexa488 (100 nM) as function of adalimumab concentration ( 0 - $1000 \mathrm{nM}$ ) in $10 \% \mathrm{v} / \mathrm{v}$ human plasma (green squares) and $20 \% \mathrm{v} / \mathrm{v}$ human plasma (red diamonds), determined by FIDA at $25^{\circ} \mathrm{C}(\mathrm{n}=3$, error bars represent standard deviation) using pre-incubated samples (> $10 \mathrm{~min}$ ). The solid lines represent fitting to the excess indicator binding isotherm (equation 7) with R2 of 0.987 (green) and 0.979 (red), respectively. 
Modes

1) Pre-incubation

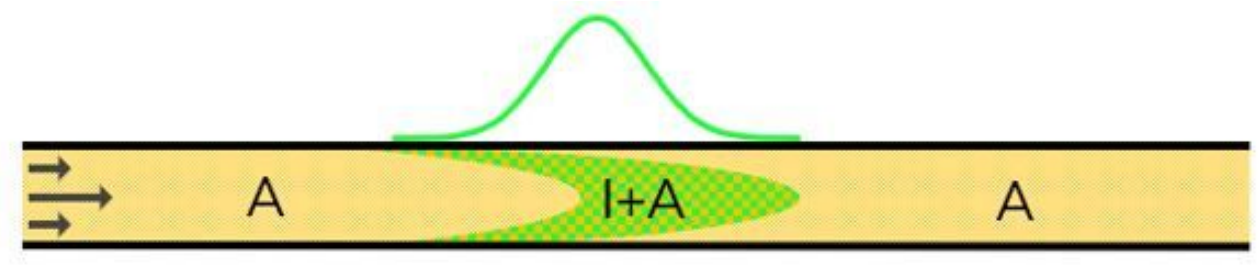

2) Capillary mix

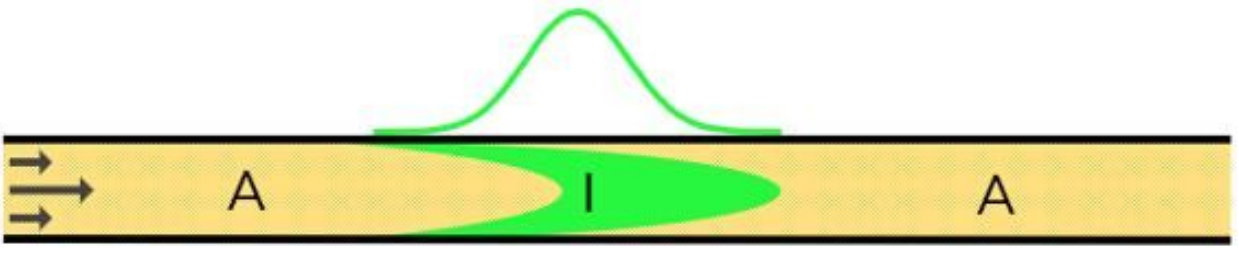

3) Complex dissociation

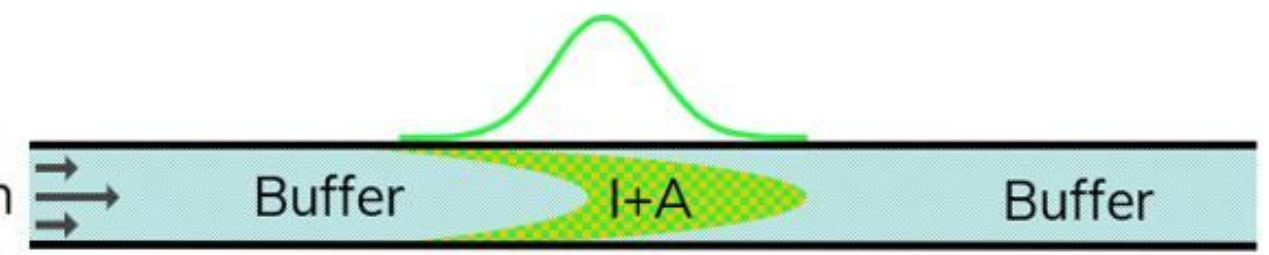

Figure 8

Schematic showing the applied incubation and mixing modes in the FIDA experiments, allowing characterization of in-solution binding kinetics.

\section{Supplementary Files}

This is a list of supplementary files associated with this preprint. Click to download.

- SupplementaryInformationfinal.docx 